\title{
Visual Estimation of Independent Motions for 3D Structures in Dynamic Environments
}

\author{
Juan Carlos Ramirez and Darius Burschka
}

Faculty for Informatics, Technische Universitaet Muenchen, Boltzmannstr. 3, Garching bei Muenchen, Germany ramirezd@in.tum.de,burschka@cs.tum.edu

\section{INTRODUCTION}

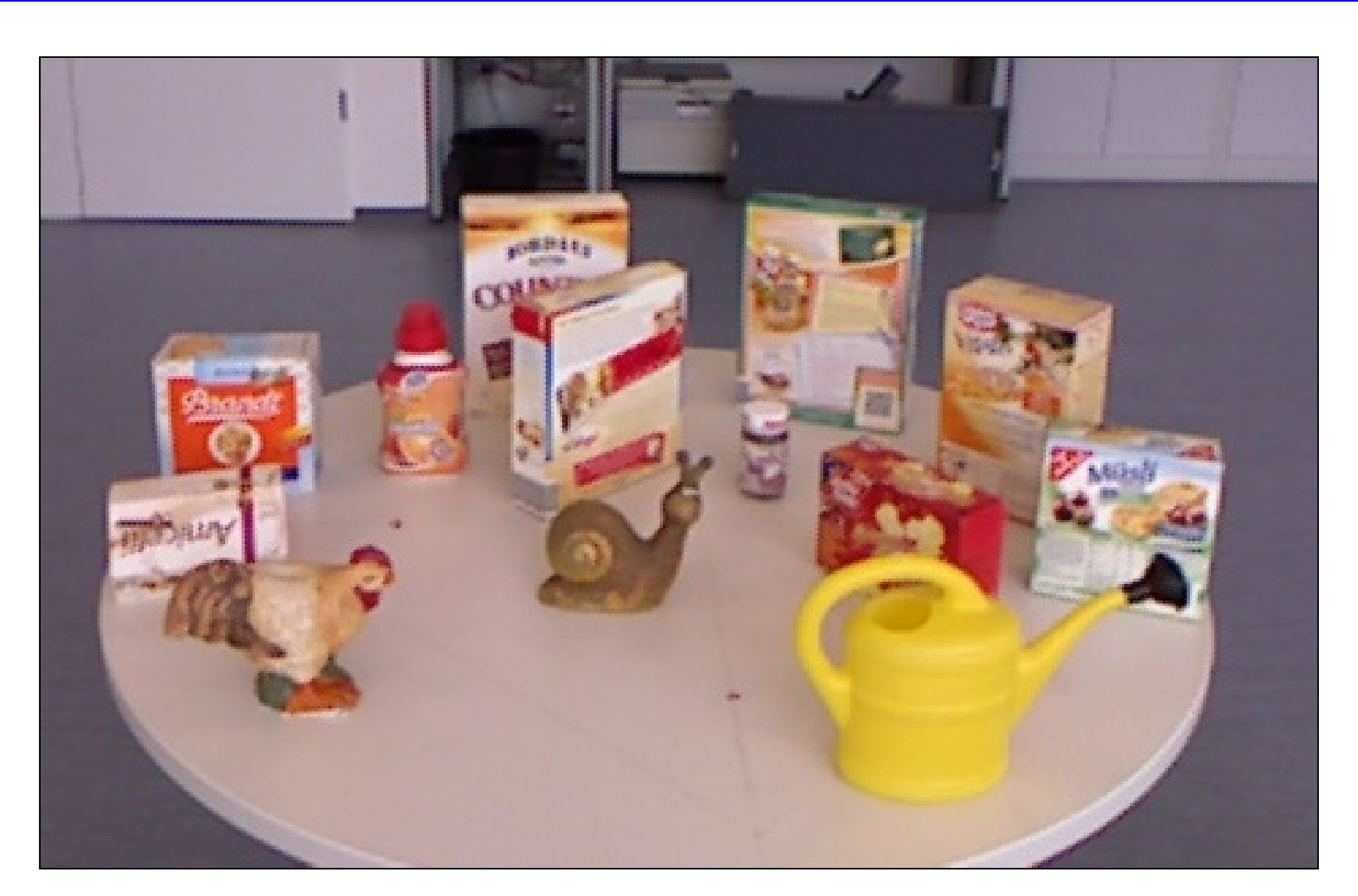

Scene

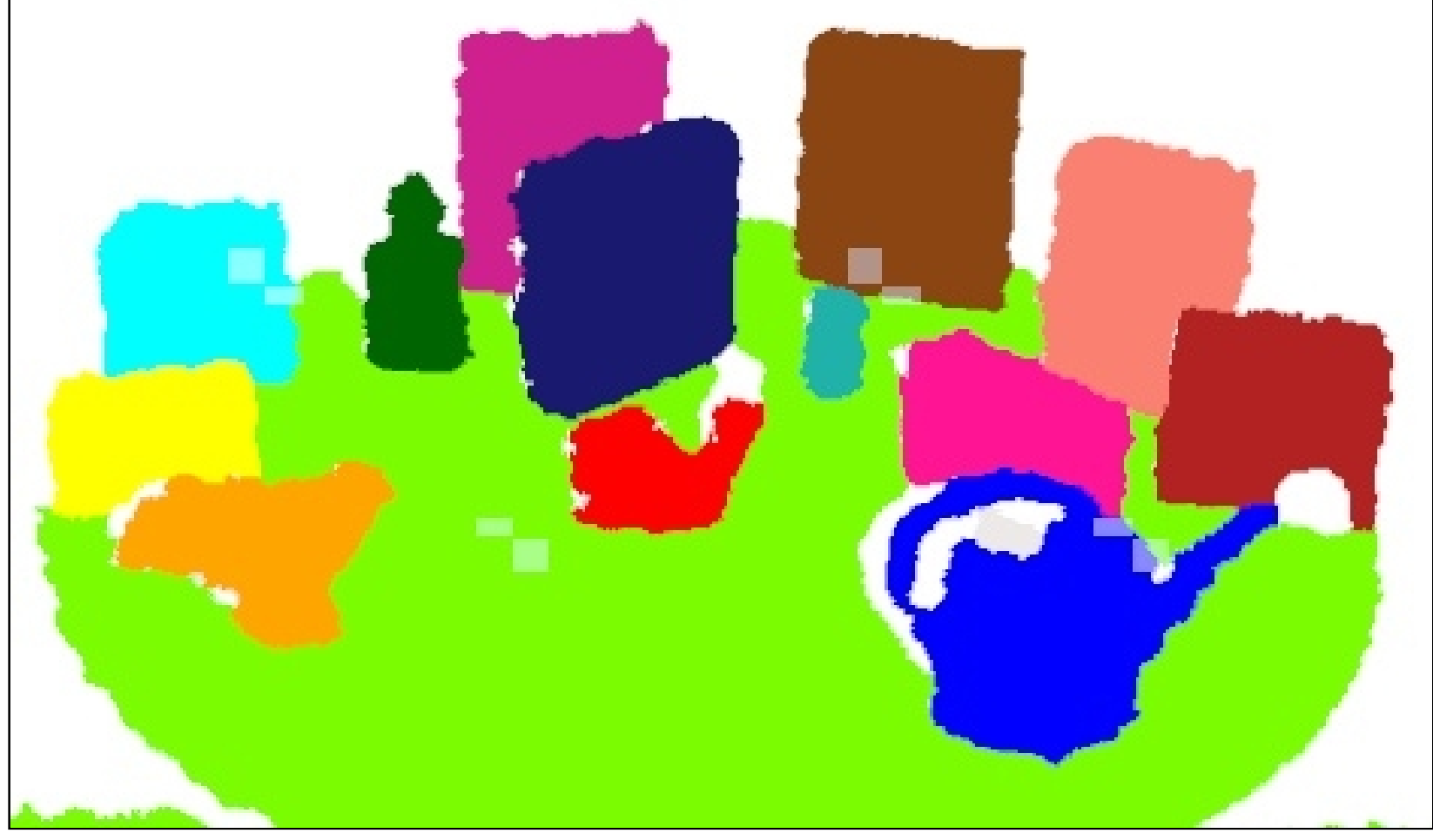

Tentative object candidates

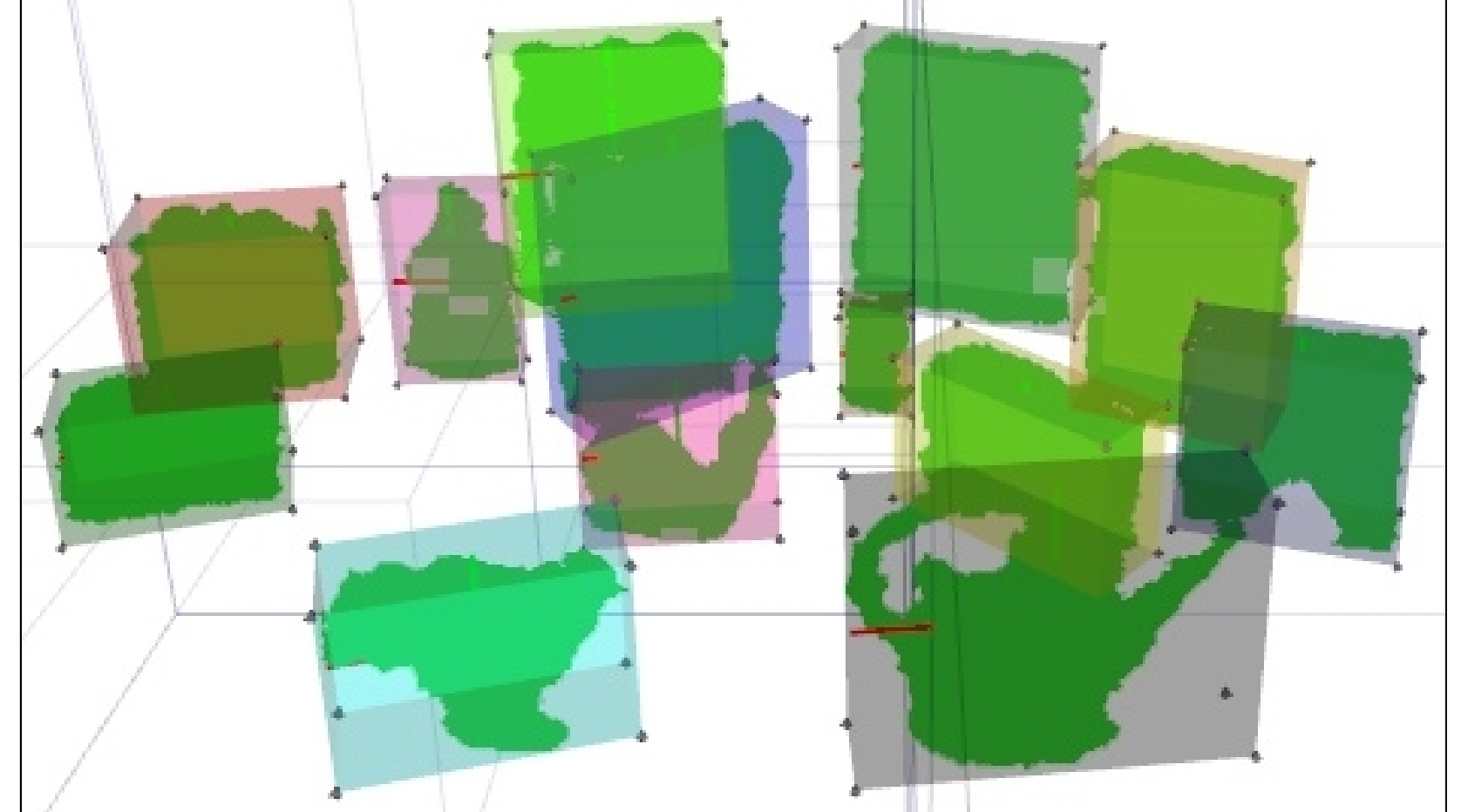

Encapsulated 3D blobs

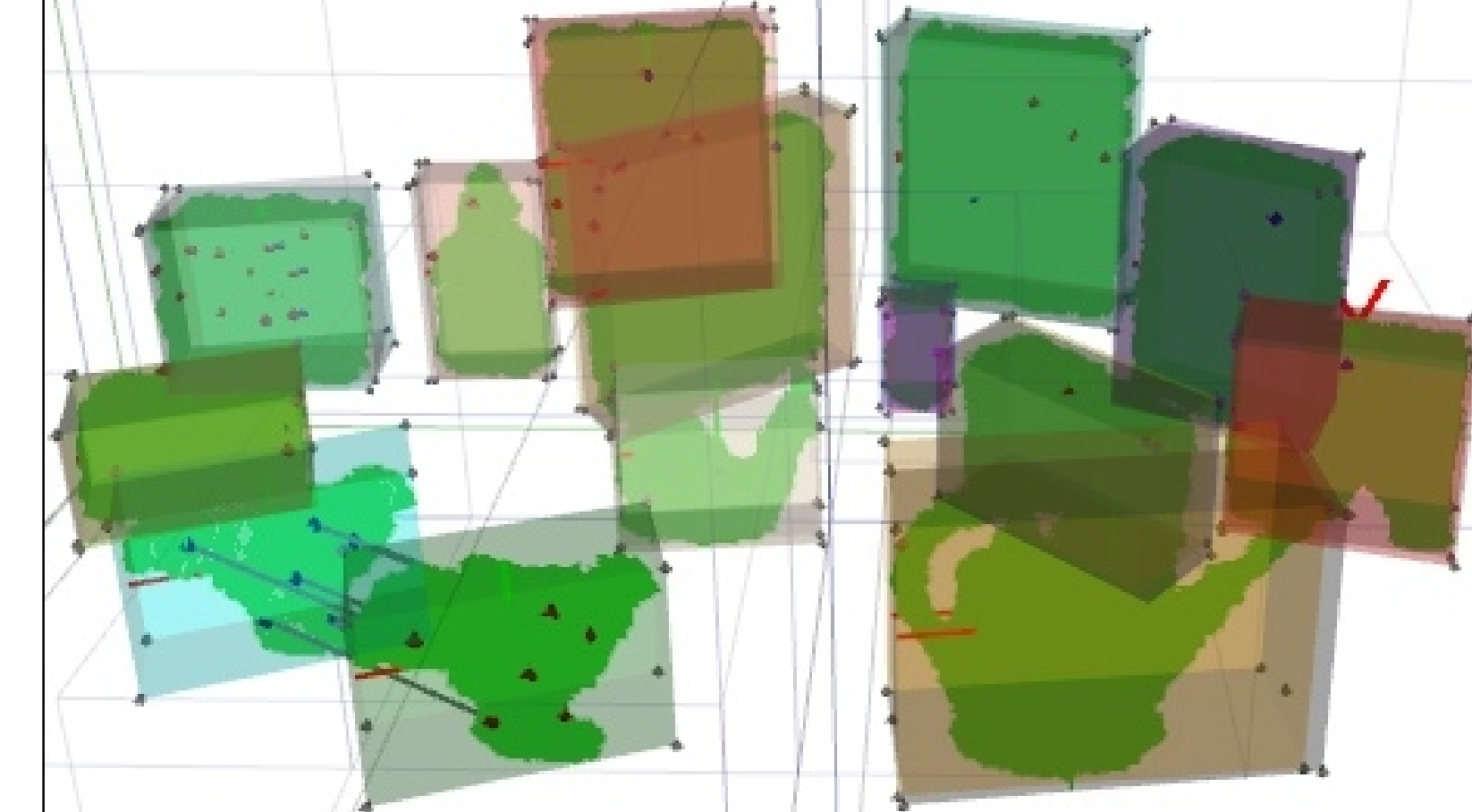

Motion estimation

An approach to consistently model and characterize potential object candidates presented in non-static scenes.

Three principal procedures support our method:

i) the segmentation of the captured range images into 3D clusters or blobs, by which we obtain a first gross

impression of the spatial structure of the scene,

ii) the maintenance and reliability of the map, which are obtained through the fusion of the captured and mapped data to which we assign a degree of existence (confidence value),

iii) the visual motion estimation of potential object candidates, through the combination of the texture and 3Dspatial information, allows not only to update the state of the actors and perceive their changes in a scene, but also to maintain and refine their individual 3D structures over time.

\section{D-MAPPING FRAMEWORK}

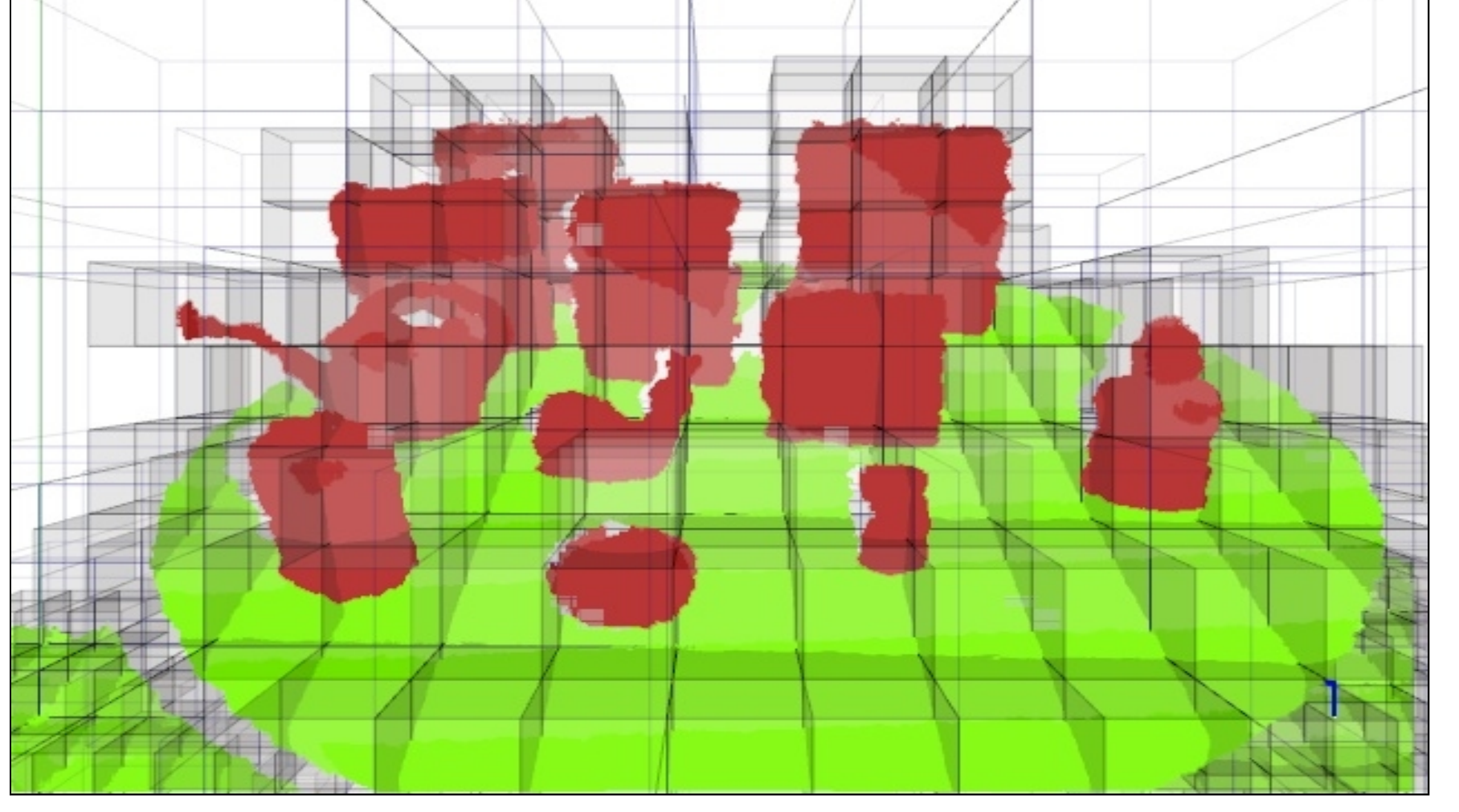

Plane detection and octree

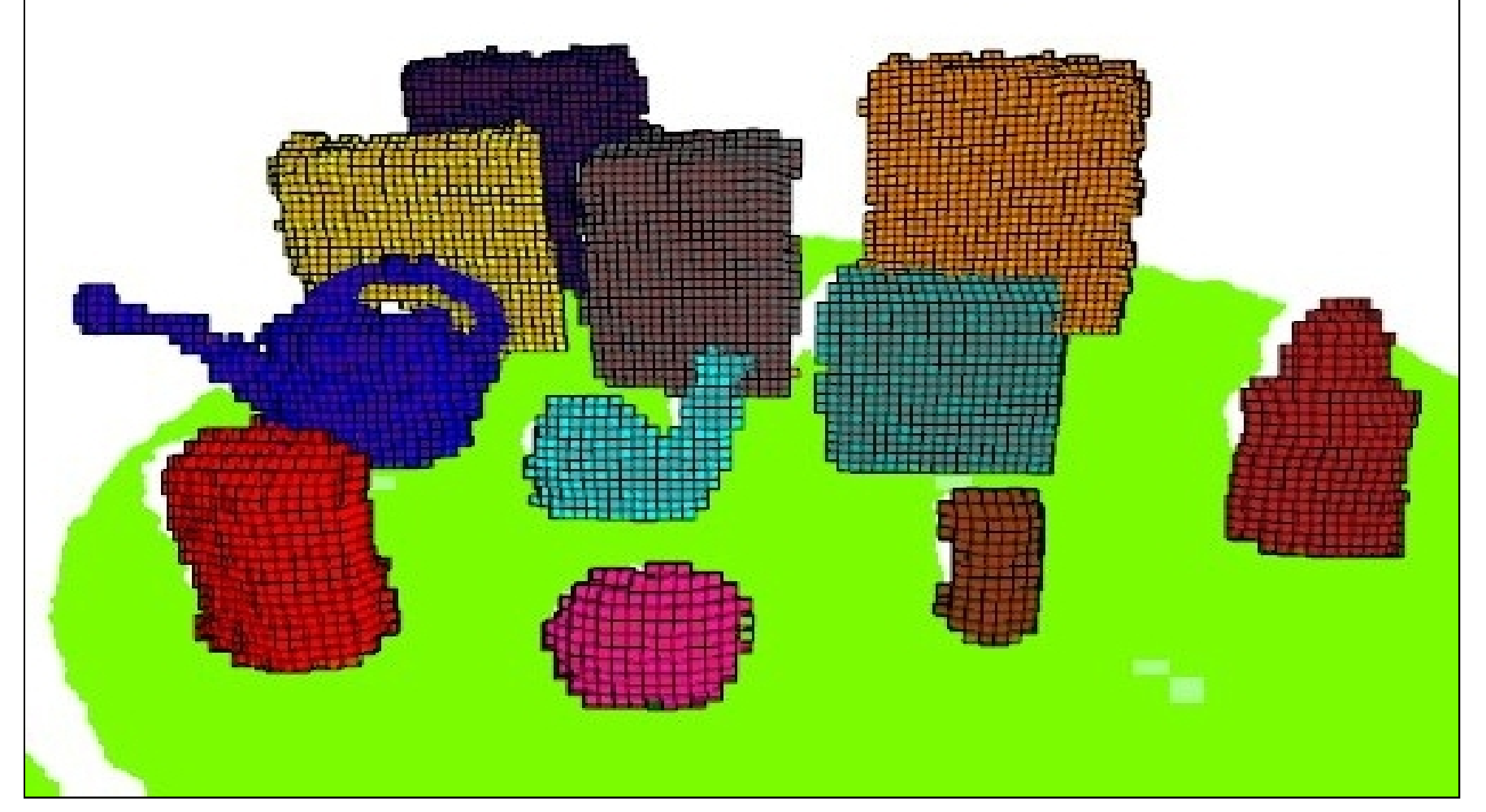

Blob detection, clustering

\section{D-Blob Detection}

After the supportingplane detection, the 3D rigid registration is stored in an octree. In order to find the spatial relations among the $3 \mathrm{D}$ points a Depth-FirstSearch (DFS) performed transversing the leaves finally identifying and clustering connected points. inside the octree and

\section{VISUAL MOTION ESTIMATION}

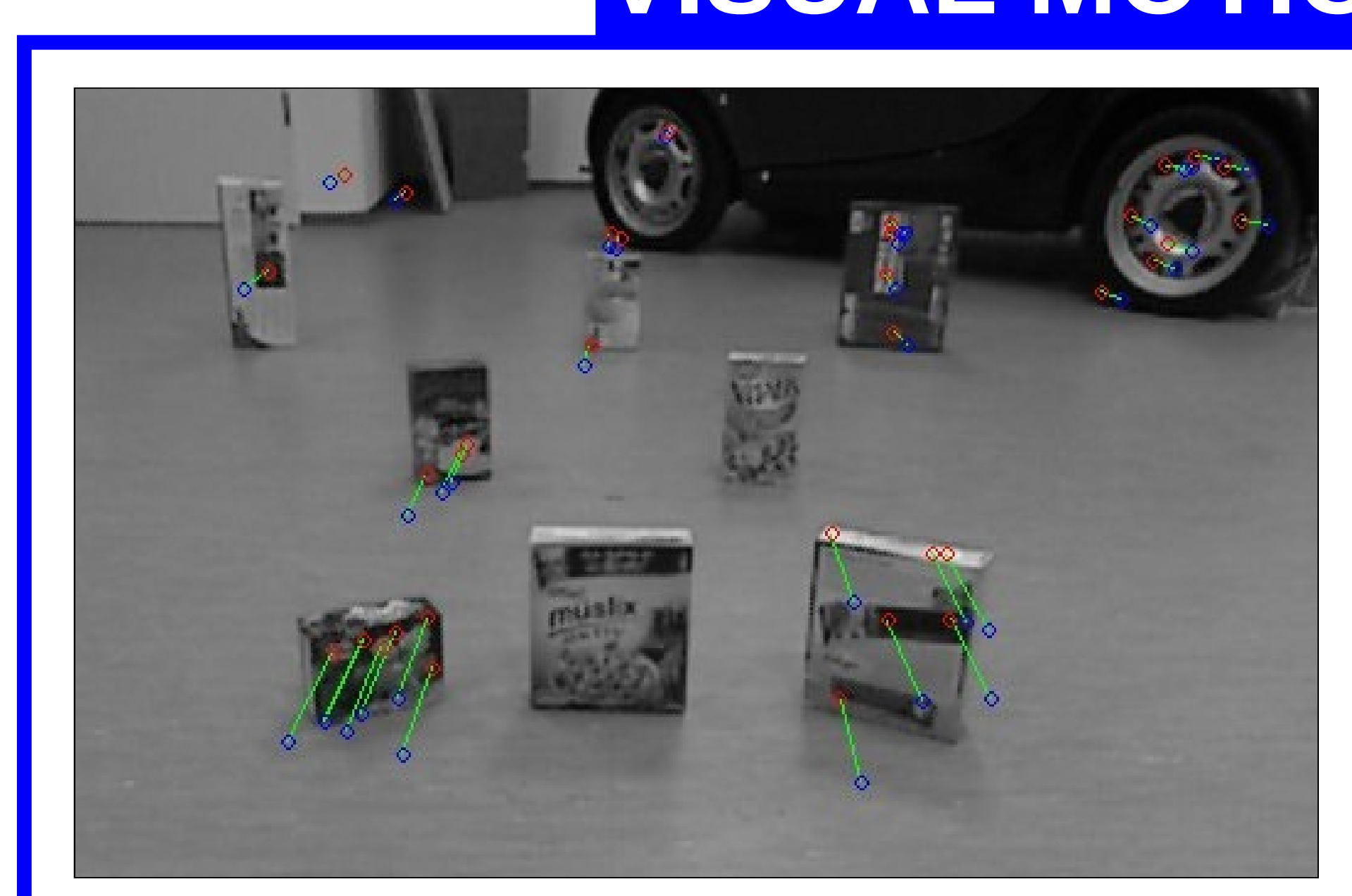

Inliers, ego-motion

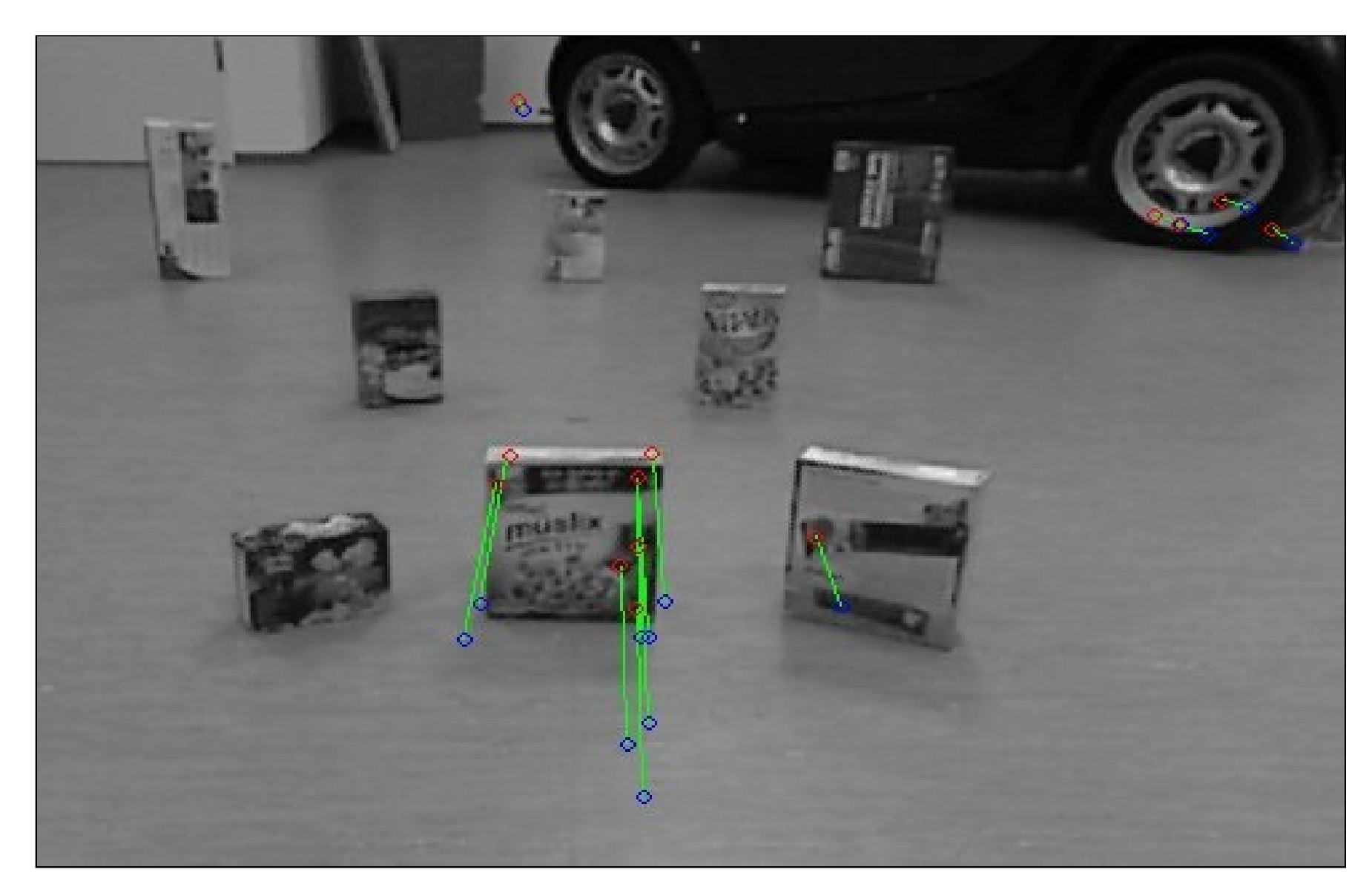

Outliers, independent-motion
Cost function:

$\Sigma^{2}=\sum_{l=1}^{L} \| p_{2, l}-\left({ }^{\text {ego }} R \cdot p_{1, l}+{ }^{\text {ego }} t\right)$

The scoring is based on the similarity of matching points:

$$
\begin{gathered}
p_{1, j}^{\prime}={ }^{\text {hyp }} R \cdot p_{1, j}{ }^{{ }^{h y p}} t \\
v_{j j}=p_{2, j}-p_{1, j} \\
x_{j}^{2}=v_{j j} S_{j}^{-1} v_{j j}^{T}<X_{\alpha}^{2} \\
S_{j}=P_{1, j}+P_{2, j}
\end{gathered}
$$

\section{EXPERIMENTS AND RESULTS}

On a wheeled robot:

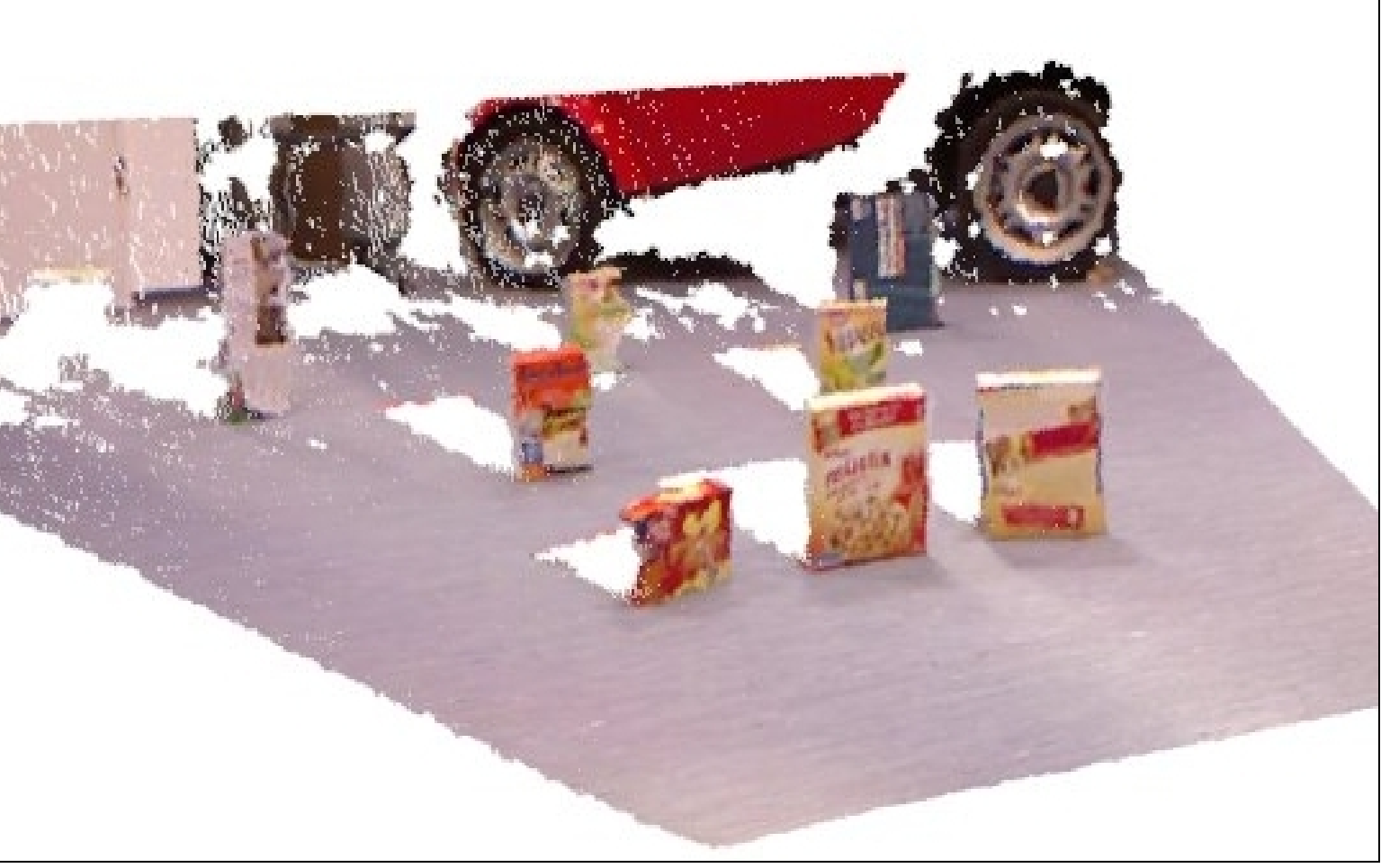

3D textured image

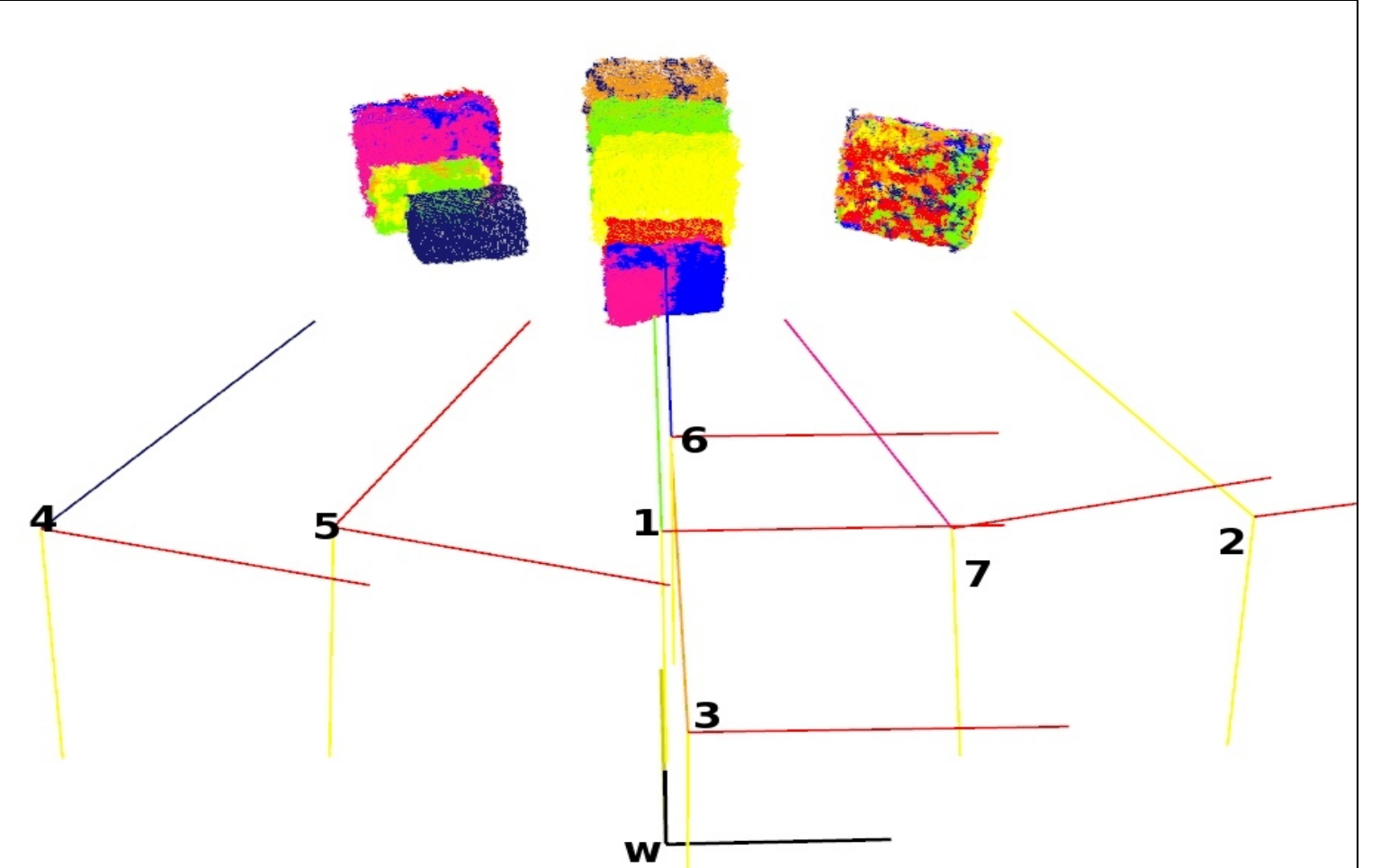

Static registrations and ego-motion detection
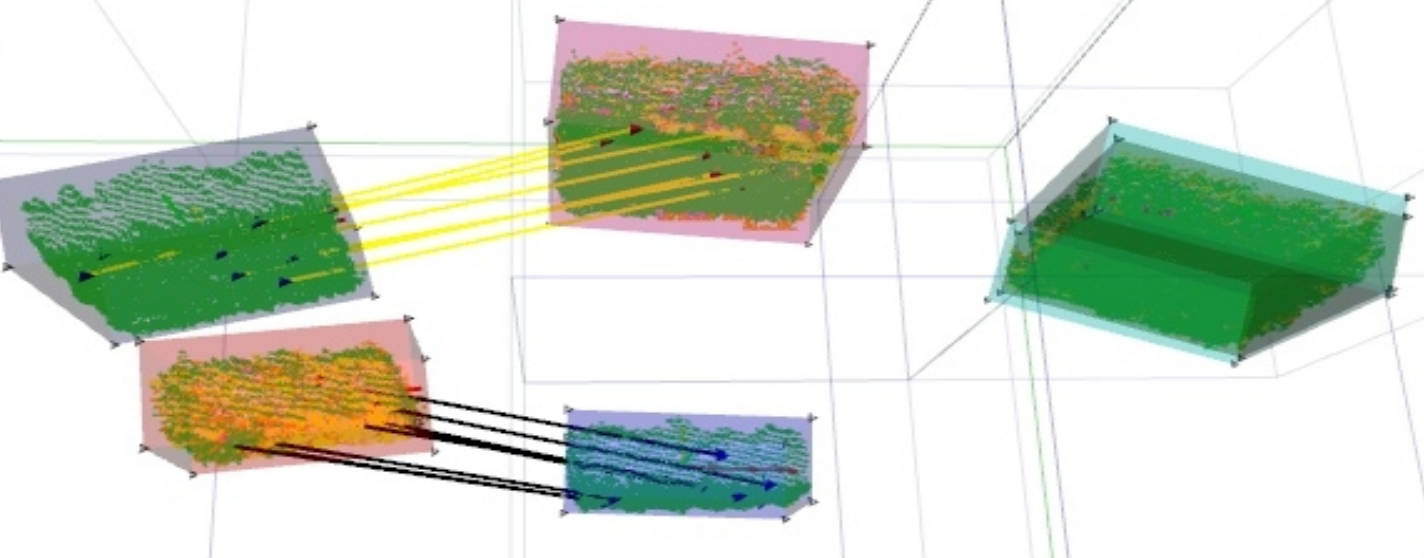

Object-motion detection in the map
In a table scene:

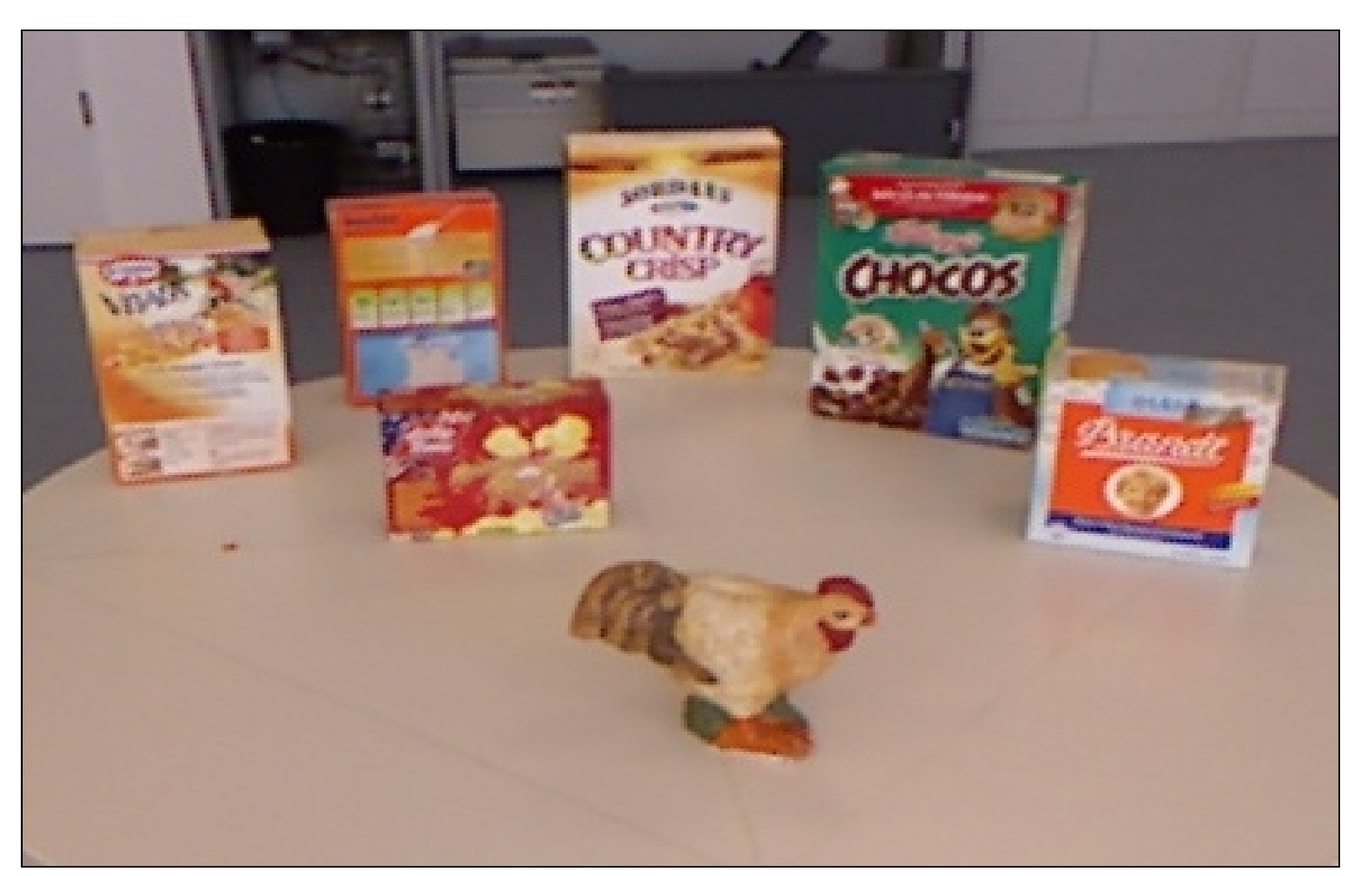

Scene

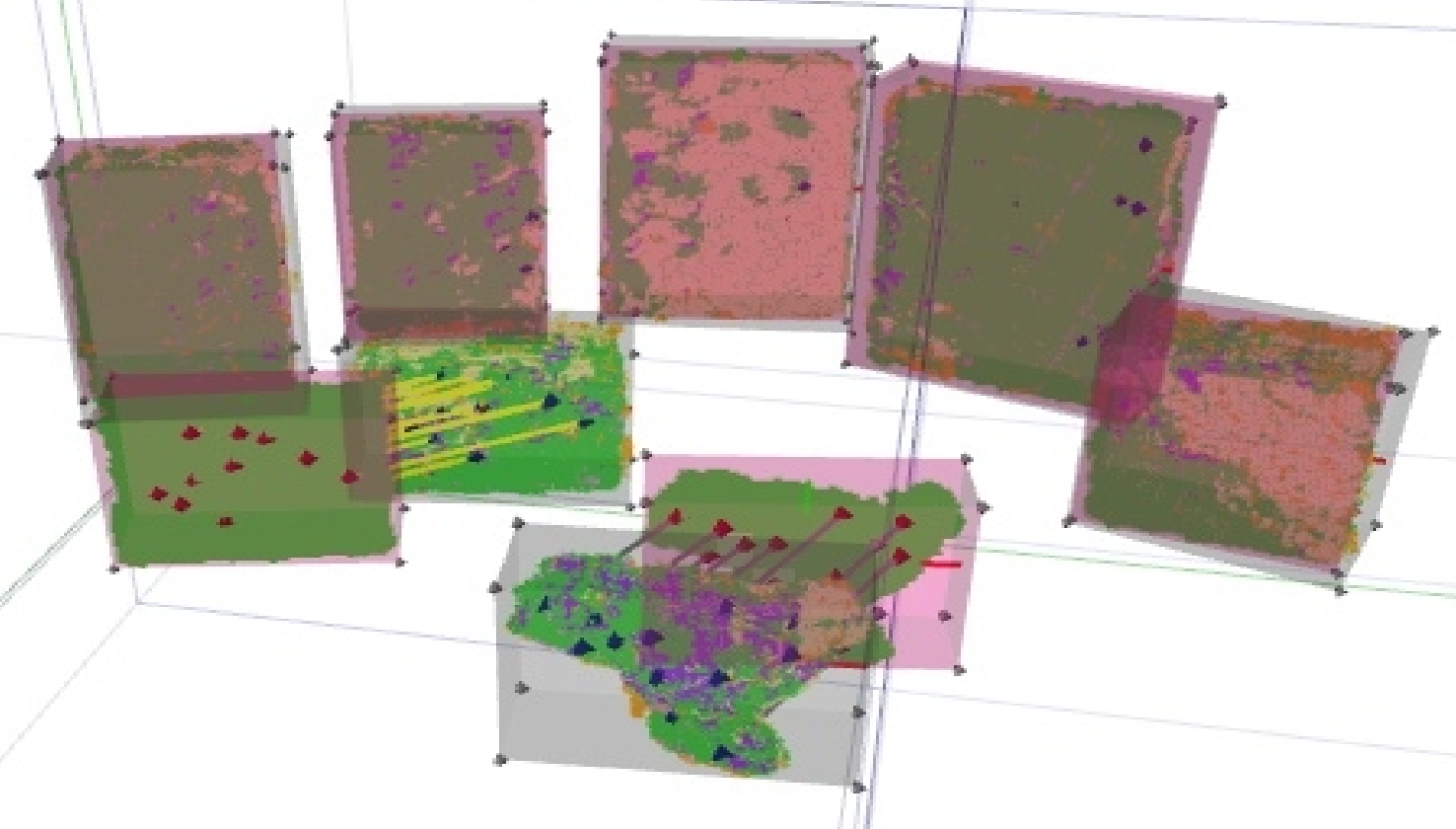

Object- and ego-motion detection

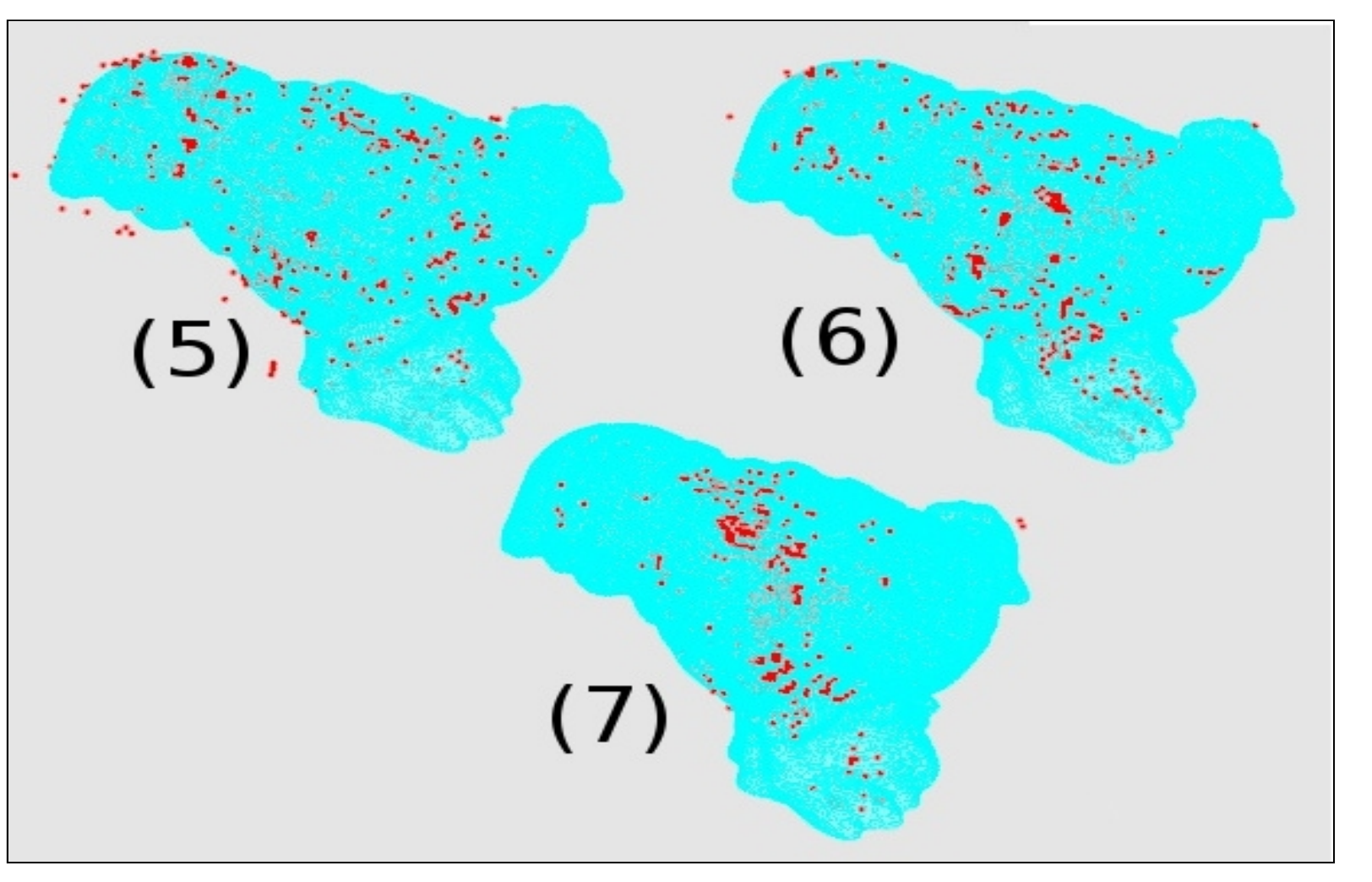

Confidence value points (red) and object model
[1] Arun, K. S., Huang, T. S., and Blostein, S. D. (1987). Least squares fitting of two 3-d point sets. IEEE Trans. Pattern Anal. Mach. Intell.
Kitt, B., Geiger, A., and Lategahn, H. (2010). Visual odometry based on stereo image sequences with ransac based outlier rejection scheme. In Intelligent Vehicles Symposium (IV), 2010 IEEE.
rate [3] Lin, K.-H. and Wang, C.-C. Stereo-based simultaneous localization, mapping and moving object tracking. In Intelligent Robots and Systems (IROS), 2010 IEEE/RSJ International Conference on. 4] Moosmann, F. and Fraichard, T. Motion estimation from range images in dynamic outdoor scenes. In Robotics and Automation (ICRA), 2010 IEEE Internation range images in dyna

5] Nister, D., Naroditsky, O., and Bergen, J. Visual odometry. In Computer Vision and Pattern

Recognition, 2004. CVPR 2004. Proceedings of the 2004 IEEE Computer Society Conference on.
Renter, [6] Ramirez, J. and Burschka, D. Framework for consistent maintenance of geometric data and abstract [6] Ramirez, J. and Burschka, D. Framework for consistent maintenance of geometric data and
task-knowledge from range observations. In Robotics and Biomimetics (ROBIO), 2011 IEEE International Conference on.

[7] Wang, C.-C., Thorpe, C., and Thrun, S. Online simultaneous localization and mapping with detection and tracking of moving objects: theory and results from a ground vehicle in crowded urban areas. In Robotics and Automation, 2003. Proceedings. ICRA '03. IEEE International Conference on. 